\title{
Generalized Euler and Class Numbers
}

\section{By Daniel Shanks}

1. Introduction. In [1] we discussed the Dirichlet series

$$
L_{a}(s)=\sum_{k=0}^{\infty}\left(\frac{-a}{2 k+1}\right)(2 k+1)^{-s}
$$

where $(-a /(2 k+1))$ is the Jacobi symbol. We defined $C_{a, n}$ and $D_{a, n}$ by

$$
L_{a}(2 n+1)=\left(\frac{\pi}{a}\right)^{2 n+1} \sqrt{ } a C_{a, n} \quad L_{-a}(2 n)=\left(\frac{\pi}{a}\right)^{2 n} \sqrt{ } a D_{a, n}
$$

and showed that these coefficients are rational for all $a=1,2,3, \cdots$ and all $n=0,1,2, \cdots$. We also showed how to compute them. We now wish to simplify these coefficients and calculations. Let

$$
\begin{gathered}
L_{a}(2 n+1)=\left(\frac{\pi}{2 a}\right)^{2 n+1} \sqrt{ } a \frac{c_{a, n}}{(2 n) !} \quad(n=0,1,2, \cdots) \\
L_{-a}(2 n)=\left(\frac{\pi}{2 a}\right)^{2 n} \sqrt{ } a \frac{d_{a, n}}{(2 n-1) !} \quad(n=1,2,3, \cdots) \text { for } a>1, \text { and } \\
L_{1}(2 n+1)=\frac{1}{2}\left(\frac{\pi}{2}\right)^{2 n+1} \frac{c_{1, n}}{(2 n) !} \quad(n=0,1,2, \cdots) \\
L_{-1}(2 n)=\frac{1}{2}\left(\frac{\pi}{2}\right)^{2 n} \frac{d_{1, n}}{(2 n-1) !} \quad(n=1,2,3, \cdots) .
\end{gathered}
$$

We now assert that the $c_{a, n}$ and $d_{a, n}$ are integers. Further, they satisfy simple recurrences on the variable $n$, and this simplifies their computation.

Consider first a short table of $c_{a, n}$ :

\begin{tabular}{r|llll}
\hline & \multicolumn{5}{|c}{$n$} \\
\cline { 2 - 5 }$a$ & 0 & 1 & \multicolumn{1}{c}{2} & \multicolumn{1}{c}{3} \\
\hline 1 & 1 & 1 & 5 & 61 \\
2 & 1 & 3 & 57 & 2763 \\
3 & 1 & 8 & 352 & 38528 \\
4 & 1 & 16 & 1280 & 249856 \\
5 & 2 & 30 & 3522 & 1066590 \\
6 & 2 & 46 & 7970 & 3487246 \\
7 & 1 & 64 & 15872 & 9493504 \\
8 & 2 & 96 & 29184 & 22880256 \\
9 & 2 & 126 & 49410 & 48649086 \\
10 & 2 & 158 & 79042 & 96448478 \\
\hline
\end{tabular}

Received March 27, 1967. 
The first row are the Euler numbers:

$$
c_{1, n}=E_{n},
$$

which are also called secant numbers since

$$
\sec w=\sum_{n=0}^{\infty} E_{n} \frac{w^{2 n}}{(2 n) !} .
$$

The first column are the class numbers; that is, there are $c_{a, 0}$ inequivalent classes of primitive binary quadratic forms

with

$$
C u^{2}+2 B u v+A v^{2}
$$

$$
A C-B^{2}=a,
$$

the principal form of which is represented by

$$
u^{2}+a v^{2} \text {. }
$$

Our two-dimensional array $c_{a, n}$ therefore generalizes both the Euler numbers and the class numbers-thus our title.

Similarly, a short table of $d_{a, n}$ is shown below. (The number $D_{a, 0}$ in (2) actually vanishes for all $a$, but we do not define $d_{a, 0}$ )

\begin{tabular}{r|llll}
\hline & \multicolumn{4}{|c}{$n$} \\
\cline { 2 - 5 }$a$ & 1 & 2 & \multicolumn{1}{c}{3} & \multicolumn{1}{c}{4} \\
\hline 1 & 1 & 2 & 16 & 272 \\
2 & 1 & 11 & 361 & 24611 \\
3 & 2 & 46 & 3362 & 515086 \\
4 & 4 & 128 & 16384 & 4456448 \\
5 & 4 & 272 & 55744 & 23750912 \\
6 & 6 & 522 & 152166 & 93241002 \\
7 & 8 & 904 & 355688 & 296327464 \\
8 & 8 & 1408 & 739328 & 806453248 \\
9 & 12 & 2160 & 1415232 & 1951153920 \\
10 & 14 & 3154 & 2529614 & 4300685074 \\
\hline
\end{tabular}

This time the first row consists of the so-called tangent numbers

$$
d_{1, n}=T_{n},
$$

since

$$
\tan w=\sum_{n=1}^{\infty} T_{n} \frac{w^{2 n-1}}{(2 n-1) !} .
$$

2. Recurrences. That these numbers are all integers follows from certain recurrences that they satisfy, and these, in turn, follow from known properties of the Euler polynomials $E_{n}(x)$. We have [2] the generator:

$$
\frac{2 e^{x t}}{e^{t}+1}=\sum_{n=0}^{\infty} E_{n}(x) \frac{t^{n}}{n !},
$$


and the known Fourier expansions:

$$
\begin{aligned}
E_{2 n}(x) & =\frac{(-1)^{n} 4(2 n) !}{\pi^{2 n+1}} S_{2 n+1}\left(\frac{x}{2}\right), \\
E_{2 n-1}(x) & =\frac{(-1)^{n} 4(2 n-1) !}{\pi^{2 n}} C_{2 n}\left(\frac{x}{2}\right),
\end{aligned}
$$

where [1, Eq. (18)]

$$
\begin{aligned}
& S_{s}(x)=\sum_{k=0}^{\infty} \frac{\sin 2 \pi(2 k+1) x}{(2 k+1)^{s}}, \\
& C_{s}(x)=\sum_{k=0}^{\infty} \frac{\cos 2 \pi(2 k+1) x}{(2 k+1)^{s}} .
\end{aligned}
$$

It follows, if we put

$$
x=2 y \text { and } t=2 v i
$$

in (9) that

$$
\begin{aligned}
& \frac{\pi}{4} \frac{\cos v(1-4 y)}{\cos v}=\sum_{n=0}^{\infty}\left(\frac{2 v}{\pi}\right)^{2 n} S_{2 n+1}(y), \\
& \frac{\pi}{4} \frac{\sin v(1-4 y)}{\cos v}=\sum_{n=1}^{\infty}\left(\frac{2 v}{\pi}\right)^{2 n-1} C_{2 n}(y) .
\end{aligned}
$$

Now, clearly,

$$
L_{1}(s)=S_{s}\left(\frac{1}{4}\right) \quad \text { and } \quad L_{-1}(s)=C_{s}(0),
$$

so that from (12) and (4), together with (6) and (8), we find that $c_{1, n}$ and $d_{1, n}$ are indeed the secant and tangent numbers, respectively.

If $a$ is divisible by a square $>1$ :

$$
a=b m^{2}
$$

with $b$ square-free, we have [1, Eq. (23)]

$$
L_{a}(s)=L_{b}(s) \prod_{p_{i} \mid m}\left[1-\left(\frac{-b}{p_{i}}\right) p_{i}^{-s}\right],
$$

the product being taken over all odd primes $p_{i}$ (if any) that divide $m$.

It follows, from (3), that

$$
\begin{aligned}
& c_{a, n}=m^{2 n}\left[m \prod_{i} p_{i}^{-1}\right]^{2 n+1} \prod_{i}\left[p_{i}{ }^{2 n+1}-\left(\frac{-b}{p_{i}}\right)\right] c_{b, n}, \\
& d_{a, n}=m^{2 n-1}\left[m \prod_{i} p_{i}^{-1}\right]^{2 n} \prod_{i}\left[p_{i}^{2 n}-\left(\frac{b}{p_{\imath}}\right)\right] d_{b, n},
\end{aligned}
$$

if $b>1$, and, from (4), that

$$
\begin{aligned}
& c_{m^{2}, n}=\frac{1}{2} m^{2 n}\left[m \prod_{i} p_{i}{ }^{-1}\right]^{2 n+1} \prod_{i}\left[p_{i}{ }^{2 n+1}-\left(\frac{-1}{p_{i}}\right)\right] c_{1, n}, \\
& d_{m^{2}, n}=\frac{1}{2} m^{2 n-1}\left[m \prod_{i} p_{i}{ }^{-1}\right]^{2 n} \prod_{i}\left[p_{i}{ }^{2 n}-1\right] d_{1, n},
\end{aligned}
$$


if $b=1$. In any case, the $c_{a, n}$ and $d_{a, n}$ are integral multiples of the $c_{b, n}$ and $d_{b, n}$, respectively.

It remains, then, to compute $c_{b, n}$ and $d_{b, n}$ for square-free $b>1$. We showed, in [1], that for such $b$ we have

$$
\begin{aligned}
L_{b}(2 n+1) & =\frac{2}{\sqrt{ } b} \sum_{k} \epsilon_{k} S_{2 n+1}\left(y_{k}\right), \\
L_{-b}(2 n) & =\frac{2}{\sqrt{ } b} \sum_{k} \epsilon_{k} C_{2 n}\left(y_{k}\right),
\end{aligned}
$$

where in the linear combinations on the right the $\epsilon_{k}$ are Jacobi symbols, and the $y_{k}$ are rational numbers, both dependent upon $b$. In all such cases, we therefore have from (12) the generators:

$$
\begin{aligned}
& \frac{\sum_{k} \epsilon_{k} \cos b w\left(1-4 y_{k}\right)}{\cos b w}=\sum_{n=0}^{\infty} w^{2 n} \frac{c_{b, n}}{(2 n) !}, \\
& \frac{\sum_{k} \epsilon_{k} \sin b w\left(1-4 y_{k}\right)}{\cos b w}=\sum_{n=1}^{\infty} w^{2 n-1} \frac{d_{b, n}}{(2 n-1) !},
\end{aligned}
$$

where we have put $v=b w$. Equating powers of $w$ gives the recurrences:

$$
\begin{aligned}
(-1)^{n} \sum_{k} \epsilon_{k}\left[b\left(1-4 y_{k}\right)\right]^{2 n} & =\sum_{i=0}^{n} c_{b, n-i}\left(-b^{2}\right)^{i}\left(\begin{array}{c}
2 n \\
2 i
\end{array}\right), \\
(-1)^{n-1} \sum_{k} \epsilon_{k}\left[b\left(1-4 y_{k}\right)\right]^{2 n-1} & =\sum_{i=0}^{n-1} d_{b, n-i}\left(-b^{2}\right)^{i}\left(\begin{array}{c}
2 n-1 \\
2 i
\end{array}\right),
\end{aligned}
$$

where the rightmost symbols are the binomial coefficients. Let us abbreviate

$$
\begin{aligned}
\sum_{i=0}^{n} c_{b, n-i}\left(-b^{2}\right)^{i}\left(\begin{array}{c}
2 n \\
2 i
\end{array}\right) & =\mathfrak{e}_{b, n}, \\
\sum_{i=0}^{n-1} d_{b, n-i}\left(-b^{2}\right)^{i}\left(\begin{array}{c}
2 n-1 \\
2 i
\end{array}\right) & =\mathscr{D}_{b, n},
\end{aligned}
$$

and note that the coefficient of $c_{b, n}\left(d_{b, n}\right)$ in these linear combinations is always 1 .

Inserting now the appropriate values of $\epsilon_{k}$ and $y_{k}$ from [1], we have the recurrences

$$
\begin{aligned}
& \mathfrak{C}_{b, n}=(-1)^{n} \sum_{k=1}^{(b-1) / 2}\left(\frac{k}{b}\right)[b-4 k]^{2 n} \quad \text { if } \quad b \equiv 3 \quad(\bmod 4), \\
& \mathfrak{e}_{b, n}=(-1)^{n} \sum_{2 k+1<b}\left(\frac{-b}{2 k+1}\right)[b-(2 k+1)]^{2 n} \quad \text { if } \quad b \neq 3 \quad(\bmod 4) \text {, } \\
& \mathbb{D}_{b, n}=(-1)^{n-1} \sum_{k=1}^{(b-1) / 2}\left(\frac{k}{b}\right)[b-4 k]^{2 n-1} \quad \text { if } \quad b \equiv 1 \quad(\bmod 4) \text {, } \\
& D_{b, n}=(-1)^{n-1} \sum_{2 k+1<b}\left(\frac{b}{2 k+1}\right)[b-(2 k+1)]^{2 n-1} \quad \text { if } \quad b \neq 1 \quad(\bmod 4) \text {. }
\end{aligned}
$$

As examples, let us list: 


$$
\begin{aligned}
\mathfrak{C}_{2, n} & =(-1)^{n} \\
\mathfrak{C}_{3, n} & =(-1)^{n} \\
\mathcal{C}_{5, n} & =(-1)^{n}\left[4^{2 n}+2^{2 n}\right] \\
\mathfrak{C}_{6, n} & =(-1)^{n}\left[5^{2 n}+1^{2 n}\right] \\
\mathfrak{C}_{7, n} & =(-1)^{n}\left[3^{2 n}+1^{2 n}-5^{2 n}\right] \\
\mathfrak{C}_{10, n} & =(-1)^{n}\left[9^{2 n}-7^{2 n}+3^{2 n}+1^{2 n}\right] \\
D_{2, n} & =(-1)^{n-1} \\
\mathscr{D}_{3, n} & =(-1)^{n-1} 2^{2 n-1} \\
\mathscr{D}_{5, n} & =(-1)^{n-1}\left[1^{2 n-1}+3^{2 n-1}\right] \\
D_{6, n} & =(-1)^{n-1}\left[5^{2 n-1}+1^{2 n-1}\right] \\
D_{7, n} & =(-1)^{n-1}\left[6^{2 n-1}+4^{2 n-1}-2^{2 n-1}\right] \\
D_{10, n} & =(-1)^{n-1}\left[9^{2 n-1}+7^{2 n-1}-3^{2 n-1}+1^{2 n-1}\right] .
\end{aligned}
$$

By such relatively simple recurrences we express $c_{b, n}\left(d_{b, n}\right)$ as a linear combination of the $c_{b, m}\left(d_{b, m}\right)$ with $m<n$, and since $c_{b, 0}$ and $d_{b, 1}$ are clearly integers, so are all of these numbers integers.

Further, for $b=1$, we have the well-known recurrences for the secant and tangent numbers, cf. [3]:

$$
\mathfrak{C}_{1, n}=0, \quad D_{1, n}=(-1)^{n-1}, \quad(n \geqq 1)
$$

and our Eqs. (22) are merely the appropriate generalization of these.

3. Comments. We have shown that the $c_{a, n}$ and $b_{a, n}$ are integers, and we have shown how they may be computed. We do not wish here to develop an elaborate theory of these numbers, and will merely close with a few brief remarks.

A. Some authors have used a notation in which the secant and tangent number coalesce into a single series, thus:

$$
c_{1, n}=E_{n}=A_{2 n}, \quad d_{1, n}=T_{n}=A_{2 n-1} .
$$

We note, from (23), that a similar joining of

or

$$
c_{2, n} \text { and } d_{2, n}
$$

$$
c_{6, n} \text { and } d_{6, n}
$$

is possible, because their recurrences fit together smoothly. But, in general, say, $a=3,5$, 7, etc., the $c_{a, n}$ and $d_{a, n}$ obey quite different laws, and therefore it does not seem desirable to attempt a joining of the complete $c_{a, n}$ and $d_{a, n}$ arrays.

B. It is clear that properties of these numbers $(\bmod m)$ may be attacked fairly generally through their recurrences (22). In a less systematic way such studies have been initiated by Glaisher [4].

C. Finally, we note that recently D. J. Newman and W. Weissblum [5] have given a combinatorial interpretation of the $A_{n}$ in

$$
\sec t+\tan t=\sum_{n=0}^{\infty} A_{n} \frac{t^{n}}{n !},
$$

where the notation here agrees with (25). They assert that $A_{n}$ is the number of "up-down" permutations of $1,2, \cdots, n$. Thus $A_{4}=c_{1,2}=5$ because 


$$
\text { 2143, 3142, 3241, 4132, and } 4231
$$

are the five ways in which 1234 may be permuted in which successive differences are alternatingly positive and negative. Presumably, reversals are not counted, e.g., 3412. This raises the question whether all of the $c_{a, n}$ and $d_{a, n}$ may not have some combinatorial interpretation.

David Taylor Model Basin

Washington, D. C. 20007

1. Daniel Shanks \& J. W. Wrench, Jr., "The calculation of certain Dirichlet series," Math. Comp., v. 17, 1963, pp. 135-154; Corrigenda, ibid., p. 488. MR 28 \#3012.

2. Milion Abramowitz \& I. A. Stegun (Editors), Handbook of Mathematical Functions, National Bureau of Standards Applied Math. Series, 55, U. S. Government Printing, Office Washington, D. C., 1964, pp. 804-805. MR 29 \#4914.

3. J. Peters, Ten-Place Logarithm Table, Vol. 1, Ungar, New York, 1957, Appendix, p. ix. (See Tafel $9 \mathrm{a}$ and $9 \mathrm{~b}$ for $T_{n}$ and $E_{n}$ to $n=30$.)

4. J. W. L. Glaisher, "The Bernoullian function," Quart. J. Pure Appl. Math., v. 29, 1898, pp. $1-168$.

5. D. J. Newman \& W. Weissblum, "Problem 67-5," SIAM Rev., v. 9, 1967, p. 121. 